\title{
EXISTENCE OF POSITIVE NONRADIAL SOLUTIONS FOR NONLINEAR ELLIPTIC EQUATIONS IN ANNULAR DOMAINS
}

\author{
SONG-SUN LIN
}

\begin{abstract}
We study the existence of positive nonradial solutions of equation $\Delta u+f(u)=0$ in $\Omega_{a}, u=0$ on $\partial \Omega_{a}$, where $\Omega_{a}=\left\{x \in \mathbb{R}^{n}: a<|x|<1\right\}$ is an annulus in $\mathbb{R}^{n}, n \geq 2$, and $f$ is positive and superlinear at both 0 and $\infty$. We use a bifurcation method to show that there is a nonradial bifurcation with mode $k$ at $a_{k} \in(0,1)$ for any positive integer $k$ if $f$ is subcritical and for large $k$ if $f$ is supercritical. When $f$ is subcritical, then a Nehari-type variational method can be used to prove that there exists $a^{*} \in(0,1)$ such that for any $a \in\left(a^{*}, 1\right)$, the equation has a nonradial solution on $\Omega_{a}$.
\end{abstract}

\section{INTRODUCTION}

In this paper we shall study the existence of positive nonradial solutions of the equation

$$
\begin{aligned}
& \Delta u+f(u)=0 \text { in } \Omega_{a}, \\
& u=0 \text { on } \partial \Omega_{a},
\end{aligned}
$$

where $\Omega_{a}=\left\{x \in \mathbb{R}^{n}: a<|x|<1\right\}$ is an annulus in $\mathbb{R}^{n}, n \geq 2$, and $f$ satisfies the following conditions:

$$
\begin{gathered}
f \in C^{1}\left(\mathbb{R}^{1}\right) \text { and } f(u)>0 \quad \text { for } u>0, \\
f(0)=0 \text { and } \lim _{u \rightarrow 0} f(u) / u=0, \\
\liminf _{u \rightarrow \infty} u f^{\prime}(u) / f(u)>1 .
\end{gathered}
$$

This paper is motivated by the work of Brezis and Nirenberg [3] and Coffman [4]. In [3], Brezis and Nirenberg proved that for any fixed domain $\Omega_{a}$, if $f(u)=u^{p}$ and $p<(n+2) /(n-2), n \geq 3$, and is near to it, then (1.1) and (1.2) has a positive nonradial solution. Later on, in [4], Coffman studied (1.1), (1.2) with $f(u)=-u+u^{p}, p>1$ and $n=2$. He proved that the number of rotationally nonequivalent positive solutions grows without bound as $a \rightarrow 1^{-}$. In both papers, problems are subcritical and variational methods are used.

In this paper, we shall use two approaches to study the problems: the bifurcation method and the variational method.

Received by the editors July 25, 1989 and, in revised form, May 21, 1990.

1980 Mathematics Subject Classification (1985 Revision). Primary 35B32, 35J65, 35P30; Secondary 34B15, 34B25.

Key words and phrases. Nonradial solution, bifurcation method, variational method.

Work partially supported by the National Science Council of the Republic of China. 
In applying the bifurcation method, we shall take a (the inner radius) as a bifurcation parameter. In spherical coordinates, the linearized equation of equations (1.1) and (1.2) at positive radial solution $u_{a}$ is

$$
\begin{gathered}
\varphi^{\prime \prime}(r)+\frac{n-1}{r} \varphi^{\prime}(r)+\left\{f^{\prime}\left(u_{a}\right)-\frac{k(k+n-2)}{r^{2}}\right\} \varphi(r) \\
=-\mu_{k, l}\left(u_{a}\right) \varphi(r), \quad a<r<1, \\
\varphi(a)=0=\varphi(1),
\end{gathered}
$$

where $k$ and $l$ are positive integers. It is well known that if there is a nonradial bifurcation at $u_{a}$, then $\mu_{k, l}\left(u_{a}\right)=0$ for some $k$ and $l$. Therefore, to look for $\mu_{k, l}=0$, it is worth knowing the signs of $\mu_{k, 1}\left(u_{a}\right)$ as $a$ approaches to 1 or 0 . We shall show that the condition (H-2) implies that, for any positive integer $k, \mu_{k, 1}\left(u_{a}\right)<0$ as $a$ approaches 1 . On the other hand, if " $u_{a}$ tends to a positive radial solution $u_{0}$ of $(1.1),(1.2)$ on the unit ball," then $\mu_{1,1}\left(u_{a}\right)>0$ as $a$ approaches 0 .

Hence, if $f$ is subcritical, i.e., $f$ satisfies

(H-3) for $u$ large, $f(u) \leq\left\{\begin{array}{l}c u^{p} \text { for some } p<\frac{n+2}{n-2} \text { if } n \geq 3, \\ \exp A(u) \text { with } A(u)=o\left(u^{2}\right) \text { at } \infty \text { if } n=2,\end{array}\right.$ then, for any $k \geq 1$, a nonradial bifurcation would occur at some $a_{k} \in(0,1)$. On the other hand, if $f$ is supercritical, i.e., $f$ satisfies

$$
u f^{\prime}(u) \geq \frac{n+2}{n-2} f(u) \text { for } u>0,
$$

we shall apply the McLeod-Serrin identity to show that $\mu_{1,1}\left(u_{a}\right) \neq 0$ for any $u_{a}$. For such $f$, we can prove that $\mu_{k, 1}\left(u_{a}\right)>0$ if $k$ is large enough. Therefore a nonradial bifurcation would also occur at some $a_{k} \in(0,1)$ when $k$ is large.

For the subcritical case, a Nehari-type variational method will also be used to study the existence of positive nonradial solutions. Indeed, consider the functionals

$$
\begin{aligned}
& J(v)=\int_{\Omega_{a}} \frac{1}{2}|\nabla v|^{2}-F(v), \\
& I(v)=\int_{\Omega_{a}}|\nabla v|^{2}-v f(v),
\end{aligned}
$$

on $H_{0}^{1}\left(\Omega_{a}\right)$, where $F(v)=\int_{0}^{v} f(t) d t$, and the numbers

$$
j(a)=\inf \left\{J(v): v \in H_{0}^{1}\left(\Omega_{a}\right) \text { and } I(v)=0\right\},
$$

and

$$
j_{\infty}(a)=\inf \left\{J(v): v \in H_{0}^{1}\left(\Omega_{a}\right), I(v)=0 \text { and } v \text { is radial }\right\} .
$$

If the minimizers of $j(a)$ and $j_{\infty}(a)$ are achieved with

$$
j(a)<j_{\infty}(a),
$$

then the minimizers of $j(a)$ will be nonradial, positive solutions of $(1.1),(1.2)$.

For $a \in(0,1)$, we can obtain (1.9) provided that all positive radial solutions of (1.1), (1.2) are "unstable with respect to nonradial modes," i.e., if $u_{a}$ is 
a positive radial solution of (1.1) and (1.2), then there exists an eigenvalue $\mu_{k, 1}\left(u_{a}\right)<0$ for some positive integer $k$. Therefore, nonradial solutions exist, provided $a$ is close to 1 .

Existence and/or uniqueness of positive radial solutions of (1.1) and (1.2) have been studied by many authors, see, e.g., Ni and Nussbaum [17], Bandle, Coffman and Marcus [1], Garaizar [5] and Lin [11]. In case $f(0)>0$, the existence of nonradial solutions has been studied by Suzuki and Nagasaki [21], Suzuki [22] and Lin [10,12].

The paper is organized as follows: In $\S 2$, we briefly discuss some properties of positive radial solutions. In $\S 3$, we study the linearized equations $(1.3)$ and (1.4) as $a \rightarrow 0^{+}$or $a \rightarrow 1^{-}$. In $\S 4$, we use the McLeod-Serrin identity to study $\mu_{k, 1}=0$. In $\S 5$, an argument of degree theory is used to show that nonradial bifurcation actually occurs at $u_{a}$ that satisfies $\mu_{k, 1}\left(u_{a}\right)=0$ and some appropriate conditions. In $\S 6$, a Nehari-type variational method is used to show that there exists a nonradial solution if $f$ is subcritical and the annuli are narrow enough.

\section{RADiAL SOLUTIONS}

In this section, we shall discuss some properties of positive radial solutions of (1.1) and (1.2) which will be used later.

A radial solution $u=u(r)$ of (1.1) and (1.2) satisfies the following equations

$$
\begin{gathered}
u^{\prime \prime}(r)+\frac{n-1}{r} u^{\prime}(r)+f(u(r))=0, \quad r \in(a, 1), \\
u(a)=0=u(1) .
\end{gathered}
$$

In Lin [11], it was proved that for any $a \in(0,1),(2.1)$ and (2.2) have a positive radial solution $u_{a}$ provided that $f$ satisfies $(\mathrm{H}-0),(\mathrm{H}-1)$ and

$$
\lim _{u \rightarrow \infty} f(u) / u=\infty \text {. }
$$

It is clear that (H-2) implies $(\mathrm{H}-2)^{\prime}$. Therefore, if $f$ satisfies $(\mathrm{H}-0) \sim(\mathrm{H}-2)$, then for any $a \in(0,1),(2.1)$ and $(2.2)$ have at least one positive radial solution.

For $n \geq 3$, set $s=r^{2-n}$ and $w(s)=u(r)$, then (2.1) and (2.2) can be written as

$$
\begin{gathered}
w^{\prime \prime}(s)+\rho(s) f(w(s))=0 \quad \text { in }\left(s_{0}, s_{1}\right), \\
w\left(s_{0}\right)=0=w\left(s_{1}\right),
\end{gathered}
$$

where $\rho(s)=(n-2)^{-2} s^{-2-\varepsilon}, \varepsilon=2 /(n-2), s_{0}=1$ and $s_{1}=a^{2-n}$. For $n=2$, set

$$
s=-\log r \text { and } w(s)=u(r),
$$

then equations $(2.1),(2.2)$ can also be written as $(2.3),(2.4)$ with $\rho(s)=e^{-2 s}$, $s_{0}=0$ and $s_{1}=-\log a$.

It is easy to check that solution $w$ of (2.3) also satisfies the following integral equation

$$
w(s)=w(\bar{s})+w^{\prime}(\bar{s})(s-\bar{s})+\int_{\bar{s}}^{s}(t-s) \rho(t) f(w(t)) d t
$$

for $s, \bar{s} \in\left(s_{0}, s_{1}\right)$.

We first study radial solutions $u_{a}$ when $a$ is close to 1 . 
Proposition 2.1. If $u_{a}$ is a solution of (2.1) and (2.2), then we have

(i) $\left\|u_{a}\right\|_{\infty} \rightarrow \infty$ as $a \rightarrow 1^{-}$,

(ii) $\int_{\Omega_{a}}\left|\nabla u_{a}\right|^{2} \rightarrow \infty$ as $a \rightarrow 1^{-}$.

Proof. Let $w(s, \alpha)$ be the solution of the following initial value problem

$$
\begin{gathered}
w^{\prime \prime}(s)+\rho(s) f(w(s))=0, \quad s>s_{0}, \\
w\left(s_{0}\right)=0 \text { and } w^{\prime}\left(s_{0}\right)=\alpha>0 .
\end{gathered}
$$

Set $s_{1}(\alpha)=\sup \left\{\bar{s}: w(s, \alpha)>0\right.$ in $\left.\left(s_{0}, \bar{s}\right)\right\}$, we claim that if $s_{1}\left(\alpha_{j}\right) \rightarrow s_{0}$ as $j \rightarrow \infty$, then $\alpha_{j} \rightarrow \infty$. We first prove that for any $\bar{s}_{1}>s_{0}$, there exists $\delta>0$ such that for any $\alpha \in(0, \delta)$,

$$
w(s, \alpha)>0 \quad \text { in }\left(s_{0}, \bar{s}_{1}\right] .
$$

In fact, if $w(s, \alpha)>0$ in $\left(s_{0}, \bar{s}_{1}\right)$, by (2.5), we have $w(s, \alpha) \leq \alpha \bar{s}_{1}$ for $s \in\left[s_{0}, \bar{s}_{1}\right]$. Now, $w$ satisfies

$$
w^{\prime \prime}(s)+\rho(s) \frac{f(w(s))}{w(s)} w(s)=0 .
$$

By (H-1) and the Sturm Comparison Theorem, (2.8) follows.

Next, we show that for any $0<m<M$,

$$
\inf \left\{s_{1}(\alpha): \alpha \in[m, M]\right\}>s_{0} .
$$

If (2.9) were false, then there would be a sequence $\left\{\alpha_{j}\right\} \subset[m, M]$ such that $\alpha_{j} \rightarrow \alpha_{0}>0$ and $s_{1}\left(\alpha_{j}\right) \rightarrow s_{1}\left(\alpha_{0}\right)=s_{0}$, a contradiction. This proves (2.9). Therefore, if $s_{1}\left(\alpha_{j}\right) \rightarrow s_{0}$ as $j \rightarrow \infty$, then $\alpha_{j} \rightarrow \infty$.

For large $\alpha$, let $\tau(\alpha) \in\left(s_{0}, s_{1}\right)$ such that $w(\tau(\alpha), \alpha)=\|w(\cdot, \alpha)\|_{\infty}$. Then by the same argument as in Lemma 2.1 of [11], we have $\lim _{\alpha \rightarrow \infty} w(\tau(\alpha), \alpha)=\infty$. This proves $(\mathrm{i})$.

(ii) Let $\tau(a) \in(a, 1)$ such that $\left\|u_{a}(\cdot)\right\|_{\infty}=u_{a}(\tau(a))$. Then

$$
\begin{aligned}
u_{a}(\tau(a)) & =\int_{a}^{\tau(a)} u^{\prime}(s) d s \leq(\tau(a)-a)^{1 / 2}\left\{\int_{a}^{\tau(a)} u^{\prime}(r)^{2} d r\right\}^{1 / 2} \\
& \leq(\tau(a)-a)^{1 / 2} a^{(1-n) / 2} \omega_{n}^{-1 / 2}\left\{\int_{\Omega_{a}}\left|\nabla u_{a}\right|^{2}\right\}^{1 / 2},
\end{aligned}
$$

where $\omega_{n}$ is the area of unit sphere $S^{n-1}$. Hence, (ii) follows.

This completes the proof.

Next, we shall study radial solutions $u_{a}$ when $a$ is close to 0 . Let $u_{\alpha} \equiv$ $u(\cdot, \alpha)$ be the solution of $(2.1),(2.2)$ with $a=a(\alpha) \in(0,1)$, and

$$
u^{\prime}(1, \alpha)=-\alpha<0 .
$$

It is easy to check that there exists a unique $\tau(\alpha) \in(a(\alpha), 1)$ such that $u(\tau(\alpha), \alpha)$ $=\|u(\cdot, \alpha)\|_{\infty}$. For such $u_{\alpha}$, define

$$
\tilde{u}_{\alpha}(r)=\tilde{u}(r, \alpha)= \begin{cases}u(r, \alpha) & \text { if } r \in[\tau(\alpha), 1], \\ u(\tau(\alpha), \alpha) & \text { if } r \in[0, \tau(\alpha)] .\end{cases}
$$


Note that $u_{0}$ is a positive radial solution of $(1.1),(1.2)$ on the unit ball $\Omega_{0}$ if it satisfies

$$
\begin{gathered}
u^{\prime \prime}(r)+\frac{n-1}{r} u^{\prime}(r)+f(u(r))=0, \quad r \in(0,1), \\
u^{\prime}(0)=0=u(1) .
\end{gathered}
$$

Proposition 2.2. Assume

(i) $u_{0}=u\left(\cdot, \alpha_{0}\right)$ is a positive radial solution on the unit ball,

(ii) there is $\delta>0$ such that for any $\alpha \in\left(\alpha_{0}, \alpha_{0}+\delta\right)\left(\right.$ or $\left.\left(\alpha_{0}-\delta, \alpha_{0}\right)\right), u_{\alpha} \equiv$ $u(\cdot, \alpha)$ is a positive radial solution on the annulus with $a=a(\alpha) \in(0,1)$ such that

$$
\left\|u_{\alpha}\right\|_{\infty} \leq M<\infty .
$$

Then, $\tilde{u}_{\alpha}$ converges uniformly to $u_{0}$ on $[0,1]$ as $\alpha \rightarrow \alpha_{0}$.

Proof. Let $\tau(\alpha) \in(a(\alpha), 1)$ such that $u(\tau(\alpha), \alpha)=\left\|u_{\alpha}\right\|_{\infty}$. We first prove that

$$
\lim _{\alpha \rightarrow \alpha_{0}} \tau(\alpha)=0
$$

If $(2.15)$ were false, there would be a sequence $\alpha_{j} \rightarrow \alpha_{0}$ and $\tau\left(\alpha_{j}\right) \rightarrow \tau_{0}>0$. Since $u^{\prime}\left(\tau\left(\alpha_{j}\right), \alpha_{j}\right)=0$, by the continuous dependence of o.d.e.'s, we have $u^{\prime}\left(\tau_{0}, \alpha_{0}\right)=0$. Since $u\left(\cdot, \alpha_{0}\right)$ is a solution on ball, by the result of Gidas, $\mathrm{Ni}$ and Nirenberg [6], $u^{\prime}\left(r, \alpha_{0}\right)<0$ on $(0,1)$, a contradiction. This proves (2.15).

Denote

$$
F(u)=\int_{0}^{u} f(s) d s
$$

and define

$$
V(r) \equiv V(r, \alpha) \equiv \frac{1}{2} u^{\prime 2}(r)+F(u(r))
$$

Since

$$
V^{\prime}(r)=-\frac{n-1}{r} u^{\prime 2}(r)<0
$$

by (2.14), we have

$$
\frac{1}{2} u^{\prime 2}(r, \alpha) \leq F(u(\tau(\alpha), \alpha)) \leq M_{1}<\infty
$$

for all $r \in[\tau(\alpha), 1]$, where $M_{1}$ is a constant. Therefore, (2.11) and (2.18) imply that

$$
\left|\tilde{u}_{\alpha}^{\prime}(r)\right| \leq\left(2 M_{1}\right)^{1 / 2} \text { on }[0,1] .
$$

Hence, by the Ascoli-Arzela Theorem, there exists a $\tilde{u} \in C([0,1])$ such that $\tilde{u}_{\alpha} \rightarrow \tilde{u}$ uniformly on $[0,1]$ as $\alpha \rightarrow \alpha_{0}$. On the other hand, by $(2.15)$, for any $r \in(0,1)$, we have $u(r, \alpha) \rightarrow u_{0}(r)$ as $\alpha \rightarrow \alpha_{0}$. Hence, $\tilde{u}=u_{0}$ on $[0,1]$.

The proof is complete.

It is not clear whether or not (2.14) always holds in Proposition 2.2. Here, we give some sufficient conditions which imply (2.14). 
Proposition 2.3. If

(i) $n \geq 3$ and there exists $\delta>0$ such that

$$
\frac{n}{n-2} f(u) \geq f^{\prime}(u) u \geq(1+\delta) f(u) \text { for } u>0,
$$

or

(2.20) $f(u)=u^{p}, 1<p<(n+2) /(n-2)$, if $n \geq 3$ and $p$ is finite if $n=2$,

then there exists $\alpha_{0}>0$ such that $u\left(\cdot, \alpha_{0}\right)$ is the unique solution on the ball and for any $\alpha \in\left(\alpha_{0}, \infty\right), u(\cdot, \alpha)$ is the unique solution on the annulus $(a(\alpha), 1)$. Moreover, there exists $M<\infty$, such that for any $\alpha \in\left(\alpha_{0}, \alpha_{0}+1\right),(2.14)$ holds. Proof. By Theorems 1.2 and 1.4 of $\mathrm{Ni}$ and Nussbaum [17], we have the first part of the theorem. By Theorem 6.6 of Bandle et al. [1], there exists a unique positive radial solution for (2.1) with the boundary condition $u^{\prime}(a)=0=u(1)$. Finally, by Theorems VII and IX of Nehari [14], (2.14) holds.

The proof is complete.

Remark 2.4. In [2], Bandle and Peletier proved that if $f(u)=u^{(n+2) /(n-2)}$, then $\left\|u_{a}\right\|_{\infty} \rightarrow \infty$ as $a \rightarrow 0^{+}$.

\section{LINEARIZED EIGENVALUE PROBLEMS}

To study the existence of nonradial solutions using bifurcation method, we need to investigate the linearized eigenvalue problem of $(1.1),(1.2)$ at positive radial solutions $u_{a}$ :

$$
\begin{aligned}
& \Delta v+f^{\prime}\left(u_{a}\right) v=-\mu v \text { in } \Omega_{a}, \\
& v=0 \text { on } \partial \Omega_{a} .
\end{aligned}
$$

In spherical coordinates, (3.1), (3.2) are reduced to

$$
\begin{gathered}
\varphi^{\prime \prime}(r)+\frac{n-1}{r} \varphi^{\prime}(r)+\left\{f^{\prime}\left(u_{a}\right)-\frac{\alpha_{k}}{r^{2}}\right\} \varphi(r) \\
=-\mu_{k, l}\left(u_{a}\right) \varphi(r), \quad a<r<1, \\
\varphi(a)=0=\varphi(1),
\end{gathered}
$$

where $\alpha_{k}=k(k+n-2), k$ and $l$ are positive integers. Note that $\alpha_{k}$ are the eigenvalues of Laplacian $-\Delta$ on $S^{n-1}$, the unit sphere, and the dimension of the eigenspace $S_{n, k}$ of associated eigenfunctions is

$$
l_{n, k}=\left(\begin{array}{c}
k+n-2 \\
k
\end{array}\right) \frac{n+2 k-2}{n+k-2} .
$$

Let $\bar{x}=\left(x_{1}, \ldots, x_{n-1}\right)$. A function $v$ defined on $S^{n-1}$ or $\Omega_{a}$ is called $O(n-1)$-invariant if $v\left(T \bar{x}, x_{n}\right)=v\left(\bar{x}, x_{n}\right)$ for all $T \in O(n-1)$. Then, for any positive integer $k$, the dimension of $V_{n, k}=\left\{v \in S_{n, k} \mid v\right.$ is $O(n-1)$ invariant $\}$ is one, for details see [19].

We first prove that if $f$ satisfies $(\mathrm{H}-2)$, then for any positive integer $k$, $\mu_{k, 1}\left(u_{a}\right)<0$ when $a$ is close to 1 . 
Lemma 3.1. If $(\mathrm{H}-0) \sim(\mathrm{H}-2)$ are satisfied. Then, for any $k \geq 1$, we have

$$
\lim _{a \rightarrow 1^{-}} \mu_{k, 1}(a)=-\infty \text {. }
$$

Proof. It is well known that $\mu_{k, 1}$ can be characterized as

$$
\mu_{k, 1}\left(u_{a}\right)=\inf _{\psi \in X_{a}} Q_{k}(\psi) / I_{2}(\psi)
$$

where

$$
\begin{gathered}
Q_{k}(\psi) \equiv Q_{k, a}(\psi) \equiv \int_{a}^{1} r^{n-1}\left\{\psi^{\prime 2}-f^{\prime}\left(u_{a}\right) \psi^{2}+\frac{\alpha_{k}}{r^{2}} \psi^{2}\right\} d r \\
I_{2}(\psi) \equiv I_{2, a}(\psi) \equiv \int_{a}^{1} r^{n-1} \psi^{2} d r
\end{gathered}
$$

and $X_{a}=H_{0}^{1}((a, 1))$.

If $u_{a}$ is a positive radial solution of $(1.1),(1.2)$, then

$$
\int_{\Omega_{a}}\left|\nabla u_{a}\right|^{2}=\int_{\Omega_{a}} u_{a} f\left(u_{a}\right) .
$$

By (H-2), there exist $\varepsilon>0$ and $M>0$ such that

$$
f^{\prime}(u) u \geq(1+\varepsilon) f(u) \quad \text { for } u \geq M .
$$

By (3.9), (3.10) and Proposition 2.1, we have

$$
\begin{aligned}
\omega_{n} Q_{k}\left(u_{a}\right) & =\omega_{n} \int_{a}^{1} r^{n-1}\left\{u_{a}^{\prime 2}-f^{\prime}\left(u_{a}\right) u_{a}^{2}+\frac{\alpha_{k}}{r^{2}} u_{a}^{2}\right\} d r \\
& =\int_{\Omega_{a}}\left\{u_{a} f\left(u_{a}\right)-f^{\prime}\left(u_{a}\right) u_{a}^{2}\right\}+\alpha_{k} \int_{\Omega_{a}} u_{a}^{2} r^{-2} \\
& \leq-\varepsilon \int_{\Omega_{a}} u_{a} f\left(u_{a}\right)+\alpha_{k} \int_{\Omega_{a}} u_{a}^{2} r^{-2}+\int_{u_{a} \leq M} u_{a} f\left(u_{a}\right)-f^{\prime}\left(u_{a}\right) u_{a}^{2} \\
& \leq-\varepsilon \int_{\Omega_{a}}\left|\nabla u_{a}\right|^{2}+\alpha_{k} a^{-2} \int_{\Omega_{a}} u_{a}^{2}+M_{1},
\end{aligned}
$$

for some constant $M_{1} \geq 0$.

Let $\nu_{1}(a)$ be the least eigenvalue of $-\Delta$ on $\Omega_{a}$ with the Dirichlet boundary condition. Then, it is easy to check that

$$
\lim _{a \rightarrow 1^{-}} \nu_{1}(a)=\infty \text {. }
$$

Using the Poincare inequality

$$
\int_{\Omega_{a}}|\nabla v|^{2} \geq \nu_{1}(a) \int_{\Omega_{a}} v^{2}
$$

for all $v \in H_{0}^{1}\left(\Omega_{a}\right)$, we obtain

$$
\omega_{n} Q_{k}\left(u_{a}\right) \leq\left\{-\varepsilon+\alpha_{k} a^{-2} \nu^{-1}(a)\right\} \int_{\Omega_{a}}\left|\nabla u_{a}\right|^{2}+M_{1} .
$$

Therefore, by using (3.11) and (3.12) again, (3.5) follows.

The proof is complete.

Next, we prove that if annulus solutions $u_{a}$ tend to a solution $u_{0}$ on the ball, in the sense of Proposition 2.2, then $\mu_{1,1}\left(u_{a}\right)>0$ as $a$ approaches 0 . 
Lemma 3.2. Under the hypotheses of Proposition 2.2. Then for any positive integer $k$,

$$
\lim _{\alpha \rightarrow \alpha_{0}} \mu_{k, 1}\left(u_{\alpha}\right)=\mu_{k, 1}\left(u_{0}\right)>0 .
$$

Proof. We first prove that $\mu_{1,1}\left(u_{0}\right)>0$. Since $u_{0}(r)$ satisfies (2.12), (2.13) with $u_{0}^{\prime}(r)<0$ in $(0,1)$, then $v\left(=-u_{0}^{\prime}\right)$ satisfies

$$
\begin{gathered}
v^{\prime \prime}(r)+\frac{n-1}{r} v^{\prime}(r)+\left\{f^{\prime}\left(u_{0}\right)-\frac{n-1}{r^{2}}\right\} v=0 \text { in }(0,1), \\
v(0)=0 \text { and } v>0 \text { in }(0,1) .
\end{gathered}
$$

Therefore, by using the Sturm Comparison Theorem, we have $\mu_{1,1}\left(u_{0}\right)>0$. Hence, $\mu_{k, 1}\left(u_{0}\right)>0$ for any positive integer $k$.

Next, we shall divide the proof of (3.13) into two parts:

(i) $\lim \sup _{\alpha \rightarrow \alpha_{0}} \mu_{k, 1}\left(u_{\alpha}\right) \leq \mu_{k, 1}\left(u_{0}\right)$,

(ii) $\liminf _{\alpha \rightarrow \alpha_{0}} \mu_{k, 1}\left(u_{\alpha}\right) \geq \mu_{k, 1}\left(u_{0}\right)$.

(i) Let $\psi_{0}>0$ be the eigenfunction associated with $\mu_{k, 1}\left(u_{0}\right)$, i.e., $Q_{k, 0}\left(\psi_{0}\right)$ $=\mu_{k, 1}\left(\psi_{0}\right)$ with the normalization $I_{2,0}\left(\psi_{0}\right)=1$. Define $\psi_{\alpha}:(a, 1) \rightarrow \mathbb{R}^{1}$, by $\psi_{\alpha}(r)=\psi_{0}((r-a) /(1-a))$, where $a=a(\alpha) \in(0,1)$.

Then

where

$$
\begin{aligned}
Q_{k, a}\left(\psi_{\alpha}\right) & =\int_{a}^{1} r^{n-1}\left\{\psi_{\alpha}^{\prime 2}-f^{\prime}\left(u_{\alpha}\right) \psi_{\alpha}^{2}+\frac{\alpha_{k}}{r^{2}} \psi_{\alpha}^{2}\right\} d r \\
& =Q_{1}(\alpha)+Q_{2}(\alpha)+Q_{3}(\alpha),
\end{aligned}
$$

$$
\begin{gathered}
Q_{1}(\alpha)=\int_{0}^{1}\left\{(1-a)^{-2} \psi_{0}^{\prime 2}(t)-f^{\prime}\left(u_{0}\right) \psi_{0}^{2}(t)\right. \\
\left.+\alpha_{k}[a+(1-a) t]^{-2} \psi_{0}^{2}(t)\right\}[a+(1-a) t]^{n-1}(1-a) d t, \\
Q_{2}(\alpha)=\int_{0}^{1} r^{n-1}\left\{f^{\prime}\left(u_{0}\right)-f^{\prime}\left(\tilde{u}_{\alpha}\right)\right\} \psi_{0}^{2}\left(\frac{r-a}{1-a}\right) d r,
\end{gathered}
$$

and

$$
Q_{3}(\alpha)=\int_{a}^{\tau(a)} r^{n-1}\left\{f^{\prime}\left(\tilde{u}_{\alpha}\right)-f^{\prime}\left(u_{\alpha}\right)\right\} \psi_{0}^{2}\left(\frac{r-a}{1-a}\right) d r .
$$

By Proposition 2.2 and (2.15), for any $\varepsilon>0$, we have $Q_{k, a}\left(\psi_{\alpha}\right) \leq \mu_{k, 1}\left(u_{0}\right)+\varepsilon$ when $\alpha$ is sufficiently close to $\alpha_{0}$.

This proves (i).

(ii) Let $\psi_{\alpha}$ be the eigenfunction associated with $\mu_{k, 1}\left(u_{\alpha}\right)$ and $I_{2, a}\left(\psi_{\alpha}\right)=1$.

Define

Then

$$
\bar{\psi}_{\alpha}(r)= \begin{cases}\psi_{\alpha}(r) & \text { if } r \in[a, 1], \\ 0 & \text { if } r \in[0, a] .\end{cases}
$$

$$
\begin{aligned}
Q_{k, 0}\left(\bar{\psi}_{\alpha}\right)= & \int_{0}^{1} r^{n-1}\left\{\bar{\psi}_{\alpha}^{\prime 2}-f^{\prime}\left(u_{0}\right) \bar{\psi}_{\alpha}^{2}+\frac{\alpha_{k}}{r^{2}} \bar{\psi}_{\alpha}^{2}\right\} d r \\
= & \int_{a}^{1} r^{n-1}\left\{\psi_{\alpha}^{\prime 2}-f^{\prime}\left(u_{\alpha}\right) \psi_{\alpha}^{2}+\frac{\alpha_{k}}{r^{2}} \psi_{\alpha}^{2}\right\} d r \\
& +\int_{a}^{1} r^{n-1}\left\{f^{\prime}\left(u_{\alpha}\right)-f^{\prime}\left(u_{0}\right)\right\} \psi_{\alpha}^{2} d r \\
= & \mu_{k, 1}\left(u_{\alpha}\right)+Q_{4}(\alpha)+Q_{5}(\alpha),
\end{aligned}
$$


where

$$
Q_{4}(\alpha)=\int_{\tau(\alpha)}^{1} r^{n-1}\left\{f^{\prime}\left(u_{\alpha}\right)-f^{\prime}\left(u_{0}\right)\right\} \psi_{\alpha}^{2} d r
$$

and

$$
Q_{5}(\alpha)=\int_{a(\alpha)}^{\tau(\alpha)} r^{n-1}\left\{f^{\prime}\left(u_{\alpha}\right)-f^{\prime}\left(u_{0}\right)\right\} \psi_{\alpha}^{2} d r
$$

We claim that

$$
\lim _{\alpha \rightarrow \alpha_{0}} \int_{a(\alpha)}^{\tau(\alpha)} r^{n-1} \psi_{\alpha}^{2}(r) d r=0
$$

Since $u_{\alpha}$ are uniformly bounded, it is easy to check that $\mu_{k, 1}\left(u_{\alpha}\right)$ are bounded, say,

$$
\left|\mu_{k, 1}\left(u_{\alpha}\right)\right| \leq C_{1}
$$

for some constant $C_{1}>0$. Therefore, by (3.6), (3.7), and (3.17), we obtain $\int_{\Omega_{a}}\left|\nabla \psi_{\alpha}\right|^{2} \leq C_{2}$, for some constant $C_{2}>0$. By the Sobolev Imbedding Theorem, we have $\int_{\Omega} \psi_{\alpha}^{2 n /(n-2)} \leq C_{3}$ for some constant $C_{3}>0$. Finally, by the Hölder inequality, we have

$$
\int_{a}^{\tau} r^{n-1} \psi_{\alpha}^{2} \leq\left\{\int_{a}^{\tau} r^{n-1}\right\}^{2 / n}\left\{\int_{a}^{\tau} \psi_{\alpha}^{2 n /(n-2)}\right\}^{(n-2) / n} \leq C_{4} \tau(\alpha)^{2}
$$

for some constant $C_{4}>0$. This proves (3.16). By (3.16) and Proposition 2.2, we have

$$
\lim _{\alpha \rightarrow \alpha_{0}} Q_{4}(\alpha)=0 \text { and } \lim _{\alpha \rightarrow \alpha_{0}} Q_{5}(\alpha)=0 .
$$

This proves (ii), and the proof is complete.

Definition 3.3. Let $u_{a}, a \in(0,1)$, be a family of positive radial solutions of (1.1) and (1.2). $u_{a}$ is called smooth in $a$ if $u_{a}$ is continuous in $a$ with respect to the $L^{\infty}$ norm.

Definition 3.4. A smooth family of positive radial solutions $u_{a}$ is said to converge to a positive radial solution $u_{0}$ on unit ball if $\tilde{u}_{a}$ converges uniformly to $u_{0}$ on $[0,1]$ as $a \rightarrow 0^{+}$, where $\tilde{u}_{a}$ is as in (2.11).

Theorem 3.5. Assume $(\mathrm{H}-0) \sim(\mathrm{H}-2)$ are satisfied. Let $u_{a}, a \in(0,1)$, be $a$ smooth family of positive radial solutions of (1.1) and (1.2) which converges to a positive radial solution $u_{0}$ on the unit ball as $a \rightarrow 0^{+}$. Then, for any $k \geq 1$, there exists $a_{k} \in(0,1)$ such that

$$
\mu_{k, 1}\left(u_{a_{k}}\right)=0 \text {. }
$$

Proof. The result follows from Lemmas 3.1 and 3.2 and the continuous dependence of eigenvalue $\mu_{k, 1}$ on $u_{a}$.

Theorem 3.6. If $f$ satisfies (2.19) or (2.20), then for any $k \geq 1$, there exists $a_{k} \in(0,1)$ such that $\mu_{k, 1}\left(u_{a_{k}}\right)=0$. Moreover, we have

$$
\mu_{k, l}\left(u_{a_{k}}\right)>0
$$

for all integers $l \geq 2$. 
Proof. By Proposition 2.3 and Theorem 3.5, we obtain the first part of the results. Since the unique radial solutions $u_{a}, a \in(0,1)$, can be obtained by a Nehari-type variational method. By Lemma 6.3 of Bandle et al. [1],

$$
\mu_{0, l}\left(u_{a}\right) \geq 0
$$

for all integers $l \geq 2$. Hence (3.19) follows from (3.20).

The proof is complete.

For supercritical case, we have the following result.

Theorem 3.7. Assume $(\mathrm{H}-0) \sim(\mathrm{H}-2)$ are satisfied. Let $u_{a}, a \in(\delta, 1)$ and $\delta \geq$ 0, be a smooth family of positive radial solutions of (1.1) and (1.2). Then, for sufficiently large $k$, there exists $a_{k} \in(\delta, 1)$ such that $\mu_{k, 1}\left(u_{a_{k}}\right)=0$.

Proof. For a fixed $a \in(\delta, 1)$, there exists $k_{0} \geq 1$ such that for any $k \geq k_{0}$, we have $\alpha_{k} / r^{2} \geq f^{\prime}\left(u_{a}\right)$ on $[a, 1]$. Hence, $\mu_{k, 1}\left(u_{a}\right)>0$ for $k \geq k_{0}$. Therefore, the result follows from Lemma 3.1.

The proof is complete.

\section{MCLeOD-SERRIN IDENTITY}

In this section we shall use the McLeod-Serrin identity [13] to study $\mu_{k, 1}=0$.

We first recall the identity and let $u$ and $\psi$ satisfy

$$
u^{\prime \prime}+\frac{n-1}{r} u^{\prime}+f(u)=0
$$

and

$$
\psi^{\prime \prime}+\frac{n-1}{r} \psi^{\prime}+g(r) \psi=0,
$$

respectively. Let

$$
Y=r^{a-b} \psi, \quad Z=\left\{r^{a}(u-c)\right\}^{\prime}, \quad W=Y Z^{\prime}-Z Y^{\prime}
$$

and $D=\left(r^{m} w\right)^{\prime}$, where $m=n-1-2 a+2 b$ and $a, b$ and $c$ are constants, then

$$
\begin{aligned}
\frac{D}{r^{m-2} Y}= & \left\{(b-1)(b+n-3)+r^{2}\left[g(r)-f^{\prime}(u)\right]\right\} Z \\
& +2 r^{a}(b-1)(a-n+2) u^{\prime} \\
& +a r^{a+1}\left\{(u-c) f^{\prime}(u)-\left(1+\frac{2 b}{a}\right) f(u)\right\} .
\end{aligned}
$$

If we choose $c=0$ and $g(r)=f^{\prime}(u)-\alpha / r^{2}$, then (4.3) can be written as

$$
\left(r^{m} W\right)^{\prime}=\left\{A r^{n-4+b} u+B r^{n-3+b} u^{\prime}+r^{n-2+b} C(u)\right\} \psi,
$$

where

$$
\begin{gathered}
A=a\{(b-1)(b+n-3)-\alpha\}, \\
B=(b-1)(b+n-3)-\alpha+2(b-1)(a-n+2), \\
C(u)=a u f^{\prime}(u)-(a+2 b) f(u)
\end{gathered}
$$

and

$$
\begin{aligned}
r^{m} W= & a(b-1) r^{n-3+b} u \psi+(a+b+1-n) r^{n-2+b} u^{\prime} \psi \\
& -a r^{n-2+b} u \psi^{\prime}-r^{n-1+b} u^{\prime} \psi^{\prime}-r^{n-1+b} f(u) \psi
\end{aligned}
$$


Furthermore, if we choose $a$ and $b$ such that $B=0$, i.e.,

$$
(b-1)(b+n-3)-\alpha+2(b-1)(a-n+2)=0,
$$

and assume that $u$ and $\psi$ satisfy

$$
u(R)=0=u(1) \text { and } \psi(R)=0=\psi(1),
$$

where $R \in(0,1)$. Then, by integrating (4.4) from $R$ to 1 , we have

$$
\begin{aligned}
& R^{n-1+b} u^{\prime}(R) \psi^{\prime}(R)-A \int_{R}^{1} r^{n-4+b} u \psi-\int_{R}^{1} r^{n-2+b} C(u) \psi \\
& =u^{\prime}(1) \psi^{\prime}(1) .
\end{aligned}
$$

By choosing appropriate $a$ and $b$, we can now prove the following result for supercritical $f$.

Theorem 4.1. Assume $f$ satisfies

$$
u f^{\prime}(u) \geq \frac{n+2}{n-2} f(u) \text { for } u>0
$$

If $u_{R}$ is a solution of (2.1) and (2.2) with $a=R$, then $\mu_{1,1}\left(u_{R}\right) \neq 0$.

Proof. Suppose $\mu_{1,1}\left(u_{R}\right)=0$. Let $\psi$ be an associated eigenfunction with $\psi>0$ in $(R, 1)$. Since $\alpha=\alpha_{1}=n-1$, it is easy to check that $a(=0)$ and $b$ (=0) satisfy (4.9) and thus $A=0$ and $C(u)=0$. Therefore, (4.11) becomes

$$
R^{n-1} u^{\prime}(R) \psi^{\prime}(R)=u^{\prime}(1) \psi^{\prime}(1) .
$$

On the other hand, $a(=n-2)$ and $b(=2)$ satisfy (4.9) too. For these choices, we have $A=0$ and $C(u)=(n-2) u f^{\prime}(u)-(n+2) f(u)$. Therefore, (4.11) becomes

$$
\begin{aligned}
R^{n+1} & u^{\prime}(R) \psi^{\prime}(R)-\int_{R}^{1} r^{n}\left\{(n-2) u f^{\prime}(u)-(n+2) f(u)\right\} \psi \\
& =u^{\prime}(1) \psi^{\prime}(1) .
\end{aligned}
$$

Therefore, if $f$ satisfies (H-4), then (4.12) and (4.13) lead to a contradiction.

The proof is complete.

Corollary 4.2. For $n \geq 3$ and $p \geq(n+2) /(n-2)$, let $u_{R}$ be the unique positive radial solution of

$$
\begin{gathered}
u^{\prime \prime}+\frac{n-1}{r} u^{\prime}+u^{p}=0 \quad \text { in }(R, 1), \\
u(R)=0=u(1) .
\end{gathered}
$$

Then $\mu_{1,1}\left(u_{R}\right)<0$ and $\mu_{k, l}\left(u_{R}\right)>0$ for $k \geq 1$ and $l \geq 2$.

Proof. By Theorem 4.1, $\mu_{1,1}\left(u_{R}\right) \neq 0$. By Lemma 3.1, $\mu_{1,1}\left(u_{R}\right)<0$ for $R$ close to 1 . Hence $\mu_{1,1}\left(u_{R}\right)<0$ for all $R \in(0,1)$. By $(3.20)$, we have $\mu_{k, l}\left(u_{R}\right)>0$ for all $k \geq 1$ and $l \geq 2$.

The proof is complete.

Also the McLeod-Serrin identity has to do with $\mu_{k, 1} \neq 0$ for $k \geq 2$. As an example, we prove the following results. 
Theorem 4.3. For $n \geq 3$ and $p>1+2(k+1) /(n-2)$, let $u_{R}$ be the solution of (4.14) and (4.15). Then $\mu_{k, 1}\left(u_{R}\right)<0$ if

$$
R \geq R(k, p, n)=\{2(k-1) /[(n-2)(p-1)-2(k+1)]\}^{1 / 2 k} .
$$

Proof. For any fixed $\alpha$, in equation (4.9), $a$ can be solved in terms of $b$; in fact,

$$
a=\frac{n-1-b}{2}+\frac{\alpha}{2(b-1)}, \quad \text { for } b \neq 1 .
$$

Let $\alpha=\alpha_{k}=k(k+n-2)$. Then

$$
A=-2\{b-(k+1)\}\{b+(k+n-3)\}\{b-(n+k-1)\}\{b+k-1\} /(b-1)
$$

and

$$
C(u)=c u^{p}, \quad \text { where } c=a(p-1)-2 b .
$$

Choosing $b_{1}=-(k-1)$ and $b_{2}=(k+1)$, we have $A_{1}=A_{2}=0, c_{1}=2(k-1)$ and $c_{2}=(n-2)(p-1)-2(k+1)$. By (4.10) and (4.11), we have

$$
\begin{aligned}
R^{n-k} & u^{\prime}(R) \psi^{\prime}(R)-c_{1} \int_{R}^{1} r^{n-k-1} u^{p} \psi \\
= & R^{n+k} u^{\prime}(R) \psi^{\prime}(R)-c_{2} \int_{R}^{1} r^{n+k-1} u^{p} \psi,
\end{aligned}
$$

which implies

$$
c_{1} \int_{R}^{1} r^{n-k-1} u^{p} \psi>c_{2} \int_{R}^{1} r^{n+k-1} u^{p} \psi .
$$

Therefore, if for any $r \in[R, 1]$

$$
c_{1} r^{n-k} \leq c_{2} r^{n+k}
$$

then (4.10) does not hold, i.e., $\mu_{k, 1}\left(u_{R}\right) \neq 0$. Finally, (4.16) follows from (4.18) by a straightforward computation.

The proof is complete.

Remark 4.4. For a fixed $k \geq 2, R(k, p, n) \rightarrow 0$ as $p \rightarrow \infty$. Therefore, it is of interest to know whether or not there exists a finite $p^{*}(k, n)>0$ such that $\mu_{k, 1}\left(u_{R}\right)<0$ for all $R \in(0,1)$ if $p>p^{*}(k, n)$. Note that $p^{*}(1, n)=$ $(n+2) /(n-2)$.

\section{SYMMETRY BREAKING}

In this section, we shall study the problem of nonradial bifurcation (symmetry breaking) of (1.1) and (1.2) at a positive radial solution $u_{a}$ with $\mu_{k, 1}\left(u_{a}\right)=0$, where $k \geq 1$.

To begin with, we shall take $a$ as a bifurcation parameter, (i.e., we vary domains). As for handling these problems we shall work in the Lagrangian formulation and then in the Eulerian formulation for computational purpose (see, e.g., Henry [7]).

We begin with the Lagrangian formulation. Fix a constant $c \in(0,1)$ and denote $\Omega=\Omega_{c}$. Then for any $t \in(0,1), \Omega_{t}=h_{t}(\Omega)$, where in spherical coordinates, $h_{t}$ is given by

$$
h_{t}\left(r, \theta_{1}, \ldots, \theta_{n-1}\right)=\left(1+\frac{t-1}{c-1}(r-1), \theta_{1}, \ldots, \theta_{n-1}\right), \quad r \in(c, 1) \text {. }
$$


The pull back $h_{t}^{*}: C^{m}\left(\Omega_{t}\right) \rightarrow C^{m}(\Omega)$ is defined by

$$
w(y, t) \equiv\left(h_{t}^{*} u\right)(y)=u\left(h_{t}(y)\right), \quad y \in \Omega .
$$

Then, equations (1.1), (1.2) on $\Omega_{t}$ can be rewritten as

$$
\begin{gathered}
L_{t} w+f(w)=0, \quad \text { in } \Omega, \\
w=0, \quad \text { on } \partial \Omega,
\end{gathered}
$$

where $L_{t}=h_{t}^{*} \Delta\left(h_{t}^{*}\right)^{-1}$. Moreover, (5.3) and (5.4) are equivalent to the nonlinear operator equation

$$
w(\cdot, t)-\Phi_{t}(w(\cdot, t))=0
$$

on $C_{0}^{1+\delta}(\bar{\Omega}) \times(0,1)$, where the nonlinear operator $\Phi_{t}: C_{0}^{1+\delta}(\bar{\Omega}) \times(0,1) \rightarrow$ $C_{0}^{1+\delta}(\bar{\Omega})$ is given by

$$
\Phi_{t}(w)=\Phi(w, t)=\left(-L_{t}\right)^{-1} f(w)
$$

$\delta \in(0,1)$ is a constant.

Since $\Phi_{t}$ is a compact operator on $C_{0}^{1+\delta}(\Omega) \times[a, b],[a, b] \subset(0,1)$, the method of degree theory can be applied to equation (5.5).

On the other hand, in the Eulerian formulation, let $u(x, t)$ be a positive solution of (1.1), (1.2) on $\Omega_{t}$, which is smooth in $t$. Let $v(x, t)=\partial u(x, t) / \partial t$. Then $v$ satisfies the following linearized equations of (1.1) and (1.2) at $u$ :

$$
\begin{gathered}
\Delta v(x, t)+f^{\prime}(u(x, t)) v(x, t)=0, \quad \text { in } \Omega_{t}, \\
v(x, t)+V(x, t) \cdot \nabla u(x, t)=0, \quad \text { on } \partial \Omega_{t},
\end{gathered}
$$

where in spherical coordinates,

$$
V(x, t)=\left(\frac{|x|-1}{t-1}, 0, \ldots, 0\right) .
$$

If $u(x, t)=u(|x|, t)$ is a positive radial solution of (1.1) and (1.2), let $v(x, t)$ $=\varphi(r, t) \psi\left(\theta_{1}, \ldots, \theta_{n-1}\right)$. Then (5.7) and (5.8) are reduced to

$$
\begin{gathered}
\varphi^{\prime \prime}(r, t)+\frac{n-1}{r} \varphi^{\prime}(r, t)+\left\{f^{\prime}(u)-\frac{\alpha_{k}}{r^{2}}\right\} \varphi(r, t) \\
=-\mu_{k, l} \varphi(r, t), \quad r \in(t, 1), \\
\varphi(t, t)=0=\varphi(1, t),
\end{gathered}
$$

for $k \geq 0$ and $l \geq 1$. These equations have been studied in previous sections.

We need the following terminology:

Definition 5.1. Let $u_{t}, t \in\left(a_{0}, b_{0}\right) \subset(0,1)$, be a smooth family of positive radial solutions of (1.1) and (1.2). $a \in(0,1)$ is called a nonradial bifurcation point (with respect to $\left.u_{t}\right)$ if every neighborhood of $\left(u_{a}, a\right)$ in $C_{0}^{1+\delta}(\bar{\Omega}) \times(0,1)$ contains a nonradial positive solution of (1.1) and (1.2). If $a$ is a bifurcation point and $\mu_{k, 1}\left(u_{a}\right)=0, k \geq 1$, then $a$ is called a nonradial bifurcation point with mode $k$. Similarly, $[a, b] \subset\left(a_{0}, b_{0}\right)$ is called a nonradial bifurcation interval if every neighborhood of $\left\{\left(u_{t}, t\right), t \in[a, b]\right\}$ in $C_{0}^{1+\delta}(\bar{\Omega}) \times(0,1)$ contains a nonradial positive solution of (1.1) and (1.2). In both cases, we say that $u_{t}$ has a nonradial bifurcation (or symmetry breaking) on $(0,1)$.

We shall restrict $(5.5)$ on the $O(n-1)$-invariant subspace $\left\{w \in C_{0}^{1+\gamma}(\bar{\Omega}) \times\right.$ $(0,1): w$ is $O(n-1)$-invariant $\}$, see the end of the first paragraph of $\S 3$. The following result is a variant of bifurcation theorems of Krasnosel'ski [9] or Rabinowitz [18], which was proved essentially in Lin [12]. The proof is omitted. 
Theorem 5.2. Let $u_{t}$ be the family of positive radial solutions of (1.1) and (1.2) which are smooth in $t \in\left(a_{0}, b_{0}\right) \subset(0,1)$.

If $a \in\left(a_{0}, b_{0}\right)$ and there exist $\varepsilon>0$ and $k \geq 1$ such that

(i) $\mu_{k, 1}\left(u_{a}\right)=0$ and $\mu_{k, 1}\left(u_{t}\right) \mu_{k, 1}\left(u_{t^{\prime}}\right)<0$ for $t \in(a-\varepsilon, a)$ and $t^{\prime} \in$ $(a, a+\varepsilon)$,

(ii) $\mu_{k, 2}(t)>0$ for $t \in(a-\varepsilon, a+\varepsilon)$, then $a$ is a nonradial bifurcation point with mode $k$.

Similarly, if (i) and (ii) are replaced by

(i) $\mu_{k, 1}\left(u_{t}\right)=0$ on $[a, b]$ and $\mu_{k, 1}\left(u_{t}\right) . \mu_{k, 1}\left(u_{t^{\prime}}\right)<0$ for $t \in(a-\varepsilon, a)$ and $t^{\prime} \in(b, b+\varepsilon)$,

(ii) $^{\prime} \mu_{k, 2}(t)>0$ for $t \in(a-\varepsilon, b+\varepsilon)$, then $[a, b]$ is a nonradial bifurcation interval.

Theorem 5.3. If $f$ satisfies (2.19) or (2.20), then for any $k \geq 1$, the radial solution $u_{a}$ has a nonradial bifurcation with mode $k$ on $(0,1)$. If $f(u)=u^{p}$ with $p \geq(n+2) /(n-2)$, then there exists $k^{*}(p)>1$, such that for any $k \geq$ $k^{*}(p), u_{a}$ has a nonradial bifurcation with mode $k$ on $(0,1)$.

Proof. The results follow from Theorems 3.6, 3.7 and 5.2.

\section{VARIATIONAL METHOD}

In this section, we shall use the Nehari-type variational method to study the existence of positive nonradial solution of (1.1), (1.2).

Consider the functionals

$$
J(v)=\int_{\Omega_{a}} \frac{1}{2}|\nabla v|^{2}-F(v)
$$

and

$$
I(v)=\int_{\Omega_{a}}|\nabla v|^{2}-v f(v)
$$

on $H_{0}^{1}(\Omega)$, where $F(v)=\int_{0}^{v} f(t) d t$. Let

$$
\begin{gathered}
M=\left\{v \in H_{0}^{1}\left(\Omega_{a}\right): I(v)=0\right\}, \\
M_{r}=\{v \in M: v \text { is radial }\} .
\end{gathered}
$$

Let $u_{a}$ be a positive radial solution of (1.1) and (1.2) which is unstable with respect to nonradial mode, i.e., the following conditions hold:

(U) there are eigenvalues $\mu_{1}<\mu_{2}<0$ and eigenfunctions $v_{1}=v_{1}(r)>0$ and $v_{2}=\varphi(r) \psi\left(\theta_{1}, \ldots, \theta_{n-1}\right)$ with $\varphi(r)>0$ in $(a, 1)$ and $\psi \not \equiv 0$ such that

$$
\begin{gathered}
\Delta v_{1}+f^{\prime}\left(u_{a}\right) v_{1}=-\mu_{1} v_{1} \text { in } \Omega_{a}, \\
v_{1}=0 \text { on } \partial \Omega_{a},
\end{gathered}
$$

and

$$
\begin{gathered}
\Delta v_{2}+f^{\prime}\left(u_{a}\right) v_{2}=-\mu_{2} v_{2} \text { in } \Omega_{a}, \\
v_{2}=0 \text { on } \partial \Omega_{a} .
\end{gathered}
$$

We first prove the following lemmas which generalize the results of Bandle et al. [1]. 
Lemma 6.1. Assume $f$ satisfies $(\mathrm{H}-0),(\mathrm{H}-1)$ and

$$
\text { there is } \tau>0 \text { such that } u f^{\prime}(u) \geq(1+\tau) f(u) \text { for all } u>0 \text {. }
$$

Let $u_{a}$ be a positive radial solution of $(1.1)$ and (1.2) and satisfy $(\mathrm{U})$. Then there exist an $\varepsilon>0$ and a smooth function $\delta:(-\varepsilon, \varepsilon) \rightarrow \mathbb{R}^{1}$ with $\delta(0)=\delta^{\prime}(0)=0$ such that for any $t \in(-\varepsilon, \varepsilon)$,

$$
I\left(u_{a}+\delta(t) v_{1}+t v_{2}\right)=0 .
$$

Proof. Define the function $H(\delta, t): \mathbb{R}^{2} \rightarrow \mathbb{R}^{1}$ by $H(\delta, t)=I\left(u_{a}+\delta v_{1}+t v_{2}\right)$. Then, it is easy to verify that

$$
H(\delta, 0)=\delta \int_{\Omega_{a}}\left\{f\left(u_{a}\right)-f^{\prime}\left(u_{a}\right) u_{a}\right\} v_{1}+O\left(\delta^{2}\right)
$$

as $\delta \sim 0$. Hence,

$$
\frac{\partial H}{\partial \delta}(0,0)=\int_{\Omega_{a}}\left\{f\left(u_{a}\right)-f^{\prime}\left(u_{a}\right) u_{a}\right\} v_{1}<0 .
$$

By the implicit function theorem, there exist $\varepsilon>0$ and a smooth function $\delta:(-\varepsilon, \varepsilon) \rightarrow \mathbb{R}^{1}$ with $\delta(0)=0$ such that (6.9) holds. To show $\delta^{\prime}(0)=0$, we note that

$$
\frac{\partial H}{\partial \delta}(\delta(t), t) \frac{d \delta}{d t}+\frac{\partial H}{\partial t}(\delta(t), t)=0,
$$

and as $t \sim 0$, we have

$$
\begin{aligned}
H(0, t)= & I\left(u_{a}+t v_{2}\right) \\
= & \int_{\Omega_{a}}\left|\nabla u_{a}\right|^{2}+2 t \nabla u_{a} \cdot \nabla v_{2}+t^{2}\left|\nabla v_{2}\right|^{2} \\
& -\left(u_{a}+t v_{2}\right)\left\{f\left(u_{a}\right)+f^{\prime}\left(u_{a}\right) t v_{2}+\frac{1}{2} f^{\prime \prime}\left(u_{a}\right) t^{2} v_{2}^{2}\right\}+O\left(t^{3}\right) \\
= & t \int_{\Omega_{a}} 2 \nabla u_{a} \cdot \nabla v_{2}-\left\{f\left(u_{a}\right)+u_{a} f^{\prime}\left(u_{a}\right)\right\} v_{2} \\
& +t^{2} \int_{\Omega_{a}}\left|\nabla v_{2}\right|^{2}-f^{\prime}\left(u_{a}\right) v_{2}^{2}-\frac{1}{2} f^{\prime \prime}\left(u_{a}\right) u_{a} v_{2}^{2}+O\left(t^{3}\right) \\
= & t^{2} \int_{\Omega_{a}} \mu_{2} v_{2}^{2}-\frac{1}{2} f^{\prime \prime}\left(u_{a}\right) u_{a} v_{2}^{2}+O\left(t^{3}\right),
\end{aligned}
$$

here,

$$
\int_{S^{n-1}} \psi\left(\theta_{1}, \ldots, \theta_{n-1}\right)=0
$$

has been used repeatedly. Therefore, $\partial H(0,0) / \partial t=0$. By $(6.11)$, we have $\delta^{\prime}(0)=0$.

The proof is complete.

Lemma 6.2. Assume (H-0), (H-1) and (H-2)" are satisfied. Let $u_{a}$ be a positive radial solution of (1.1) and (1.2) and satisfy $(\mathrm{U})$. Then

$$
J\left(u_{a}+\delta(t) v_{1}+t v_{2}\right)=J\left(u_{a}\right)+\frac{1}{2} \mu_{2} t^{2}+O\left(t^{4}\right)
$$


as $t \rightarrow 0$. In particular, $J\left(u_{a}\right)$ is not the infimum of $J$ over $M$.

Proof. After some calculations, we have

$$
\begin{aligned}
J\left(u_{a}\right. & \left.+\delta(t) v_{1}+t v_{2}\right)-J\left(u_{a}\right) \\
& =\frac{1}{2} \mu_{1} \delta^{2}(t) \int_{\Omega_{a}} v_{1}^{2}+\frac{1}{2} \mu_{2} t^{2} \int_{\Omega_{a}} v_{2}^{2}+O\left(t^{4}\right),
\end{aligned}
$$

here (6.12) are used. Since $\delta(0)=\delta^{\prime}(0)=0,(6.13)$ follows.

The proof is complete.

Now, we can prove the following theorem.

Theorem 6.3. Assume $(\mathrm{H}-0),(\mathrm{H}-1),(\mathrm{H}-2)^{\prime \prime}$ and $(\mathrm{H}-3)$ are satisfied. Then there exists an $a^{*} \in(0,1)$ such that for any $a \in\left(a^{*}, 1\right),(1.1)$ and (1.2) have a nonradial solution.

Proof. We note that (H-2)" implies $M_{1}=0$ in the proof of Lemma 3.1. Therefore, by Lemma 3.1 , there exists an $a^{*} \in(0,1)$ such that $\mu_{1,1}\left(u_{a}\right)<0$ for any positive radial solution $u_{a}$ of (1.1) and (1.2) with $a \in\left(a^{*}, 1\right)$. Hence, by Lemma 6.2, we have $J\left(u_{a}\right)>j(a) \equiv \inf _{v \in M} J(v)$. Since $j(a)$ is achieved by some $\bar{u}_{a} \in M$ and $\bar{u}_{a}$ is a positive solution of (1.1) and (1.2), (see, e.g., Ni [16]). Therefore, $\bar{u}_{a}$ is nonradial.

The proof is complete.

\section{REFERENCES}

1. C. Bandle, C. V. Coffman, and M. Marcus, Nonlinear elliptic problems in annular domains, J. Differential Equations 69 (1987), 322-345.

2. C. Bandle and L. A. Peletier, Nonlinear elliptic problems with critical exponent in shrinking annuli, Math. Ann. 280 (1988), 1-19.

3. H. Brezis and L. Nirenberg, Positive solutions of nonlinear elliptic equations involving critical Sobolev exponents, Comm. Pure Appl. Math. 34 (1983), 437-477.

4. C. V. Coffman, A nonlinear boundary value problem with many positive solutions, J. Differential Equations 54 (1984), 429-437.

5. X. Garaizar, Existence of positive radial solutions for semilinear elliptic equations in the annulus, J. Differential Equations 70 (1987), 69-92.

6. B. Gidas, W.-M. Ni, and L. Nirenberg, Symmetry and related properties via the maximum principle, Comm. Math. Phys. 68 (1979), 209-243.

7. D. Henry, Topics in nonlinear analysis, Trabalho de Mathematica No. 192, Universidade de Brasilia, 1982.

8. B. Kawohl, Rearrangements and convexity of level sets in PDE, Lecture Notes in Math., vol. 1150, Springer, 1985, pp. 95-97.

9. M. A. Krasnosel'skii, Topological methods in the theory of nonlinear integral equations, Pergamon, 1964.

10. S. S. Lin, On non-radially symmetric bifurcation in the annulus, J. Differential Equations 80 (1989), 251-279.

11. _ On the existence of positive radial solutions for nonlinear elliptic equations in annular domains, J. Differential Equations 81 (1989), 221-233.

12. _ Positive radial solutions and non-radial bifurcation for semilinear elliptic equations in annular domains, J. Differential Equations 86 (1990), 367-391.

13. K. McLeod and J. Serrin, Uniqueness of positive radial solutions of $\Delta u+f(u)=0$ in $\mathbb{R}^{n}$, Arch. Rational Mech. Anal. 99 (1987), 115-145.

14. Z. Nehari, On a class of nonlinear second order differential equations, Trans. Amer. Math. Soc. 95 (1960), 101-123. 
15. W.-M. Ni, Some aspects of semilinear elliptic equations, Lecture Notes, National Tsing Hua University, Hsinchu, Taiwan, May 1987.

16. __ Recent progress in semilinear elliptic equations, Solutions for Nonlinear Elliptic Equations (T. Suzuki, ed.), RIMS Kokyuroku 679, Research Institute for Mathematical Science, Kyoto University, Kyoto, Japan, February, 1989.

17. W.-M. Ni and R. Nussbaum, Uniqueness and non-uniqueness for positive radial solution of $\Delta u+f(u, r)=0$, Comm. Pure Appl. Math. 38 (1985), 67-108.

18. P. H. Rabinowitz, Some global results for nonlinear eigenvalue problems, J. Functional Analysis 7 (1971), 487-513.

19. J. Smoller and A. Wasserman, Symmetry-breaking for solution of semilinear elliptic equations with general boundary conditions, Comm. Math. Phys. 105 (1986), 415-441.

20. T. Suzuki and K. Nagasaki, On the nonlinear eignevalue problem $\Delta u+\lambda e^{u}=0$, Trans. Amer. Math. Soc. 309 (1988), 591-608.

21. __ Lifting of local subdifferentiations and elliptic boundary value problems on symmetric domains. I, Proc. Japan Academy 64 (1988), 1-4.

22. T. Suzuki, Symmetric domains and elliptic equations, preprint.

Department of Applied Mathematics, National Chiao Tung University, Hsin-chu, TaIWAN, REPUBLIC OF CHINA

E-mail address: sslin@twnctu01.bitnet 